\title{
Neutron cross-sections for advanced nuclear systems: the n_TOF project at CERN
}

M. Barbagallo ${ }^{1, a}$, M. Mastromarco ${ }^{1}$, N. Colonna ${ }^{1}$, S. Altstadt ${ }^{2}$, J. Andrzejewski ${ }^{3}$, L. Audouin ${ }^{4}$, V. Bécares ${ }^{5}$, F. Bečváŕ ${ }^{6}$, F Belloni ${ }^{7}$, E. Berthoumieux ${ }^{7,8}$, J. Billowes ${ }^{9}$, D. Bosnar ${ }^{10}$, M. Brugger ${ }^{8}$, M. Calviani ${ }^{8}$, F. Calviño ${ }^{11}$, D. Cano-Ott ${ }^{5}$, C. Carrapiço ${ }^{12}$, F. Cerutti ${ }^{8}$, E. Chiaveri ${ }^{7,8}$, M. Chin ${ }^{8}$, G. Cortés ${ }^{11}$, M.A. Cortés-Giraldo ${ }^{13}$, M. Diakaki ${ }^{14}$, C. Domingo-Pardo ${ }^{15}$, I. Duran ${ }^{16}$, R. Dressler ${ }^{17}$, C. Eleftheriadis ${ }^{18}$, A. Ferrari ${ }^{8}$, K. Fraval ${ }^{7}$, S. Ganesan ${ }^{19}$, A.R. García ${ }^{5}$, G. Giubrone ${ }^{15}$, I.F. Gonçalves ${ }^{12}$, E. González-Romero ${ }^{5}$, E. Griesmayer ${ }^{20}$, C. Guerrero ${ }^{8}$, F. Gunsing ${ }^{7}$, A. Hernández-Prieto ${ }^{8,11}$, D.G. Jenkins ${ }^{21}$, E. Jericha ${ }^{20}$, Y. Kadi ${ }^{8}$, F. Käppeler ${ }^{22}$, D. Karadimos ${ }^{14}$, N. Kivel ${ }^{17}$, P. Koehler ${ }^{23}$, M. Krtička ${ }^{6}$, J. Kroll ${ }^{6}$, C. Lampoudis ${ }^{7}$, C. Langer ${ }^{2}$, E. Leal-Cidoncha ${ }^{16}$, C. Lederer ${ }^{24}$, H. Leeb ${ }^{20}$, L.S. Leong ${ }^{4}$, R. Losito ${ }^{8}$, A. Manousos ${ }^{18}$, J. Marganiec $^{3}$, T. Martínez ${ }^{5}$, C. Massimi ${ }^{25}$, P.F. Mastinu ${ }^{26}$, E. Mendoza ${ }^{5}$, A. Mengoni' ${ }^{27}$, P.M. Milazzo ${ }^{28}$, F. Mingrone ${ }^{25}$, M. Mirea ${ }^{29}$, W. Mondalaers ${ }^{30}$, C. Paradela ${ }^{16}$, A. Pavlik ${ }^{24}$, J. Perkowski $^{3}$, A. Plompen ${ }^{30}$, J. Praena $^{13}$, J.M. Quesada ${ }^{13}$, T. Rauscher ${ }^{31}$, R. Reifarth ${ }^{2}$, A. Riego ${ }^{11}$, C. Rubbia ${ }^{8}$, M. Sabaté-Gilarte ${ }^{13}$, R. Sarmento ${ }^{12}$, A. Saxena ${ }^{19}$, P. Schillebeeckx ${ }^{30}$, S. Schmidt ${ }^{2}$, D. Schumann ${ }^{17}$, G. Tagliente ${ }^{1}$, J.L. Tain ${ }^{15}$, D. Tarrío ${ }^{16}$, L. Tassan-Got ${ }^{4}$, A. Tsinganis ${ }^{8}$, S. Valenta ${ }^{6}$, G. Vannini2 ${ }^{25}$, V. Variale ${ }^{1}$, P. Vaz ${ }^{12}$, A. Ventura ${ }^{27}$, M.J. Vermeulen ${ }^{21}$, V. Vlachoudis ${ }^{8}$, R. Vlastou ${ }^{14}$, A. Wallner ${ }^{24}$, T. Ware ${ }^{9}$, M. Weigand ${ }^{2}$, C. Weiß ${ }^{20}$, T. Wright ${ }^{9}$ and P. Žugec ${ }^{10}$

${ }^{1}$ Istituto Nazionale Fisica Nucleare, Sezione di Bari, V. Orabona 4, 70125 Bari, Italy

${ }^{2}$ Johann-Wolfgang-Goethe Universität, Frankfurt, Germany

${ }^{3}$ Uniwersytet Łódzki, Lodz, Poland

${ }^{4}$ Centre National de la Recherche Scientifique/IN2P3 - IPN, Orsay, France

${ }^{5}$ Centro de Investigaciones Energeticas Medioambientales y Tecnológicas (CIEMAT), Madrid, Spain

${ }^{6}$ Charles University, Prague, Czech Republic

${ }^{7}$ Commissariat à l'Énergie Atomique (CEA) Saclay - Irfu, Gif-sur-Yvette, France

${ }^{8}$ European Organization for Nuclear Research (CERN), Geneva, Switzerland

${ }^{9}$ University of Manchester, Oxford Road, Manchester, UK

${ }^{10}$ Department of Physics, Faculty of Science, University of Zagreb, Croatia

${ }^{11}$ Universitat Politecnica de Catalunya, Barcelona, Spain

${ }^{12}$ Instituto Tecnológico e Nuclear, Instituto Superior Técnico, Universidade Técnica de Lisboa, Lisboa, Portugal

${ }^{13}$ Universidad de Sevilla, Spain

${ }^{14}$ National Technical University of Athens (NTUA), Greece

${ }^{15}$ Instituto de Física Corpuscular, CSIC-Universidad de Valencia, Spain

${ }^{16}$ Universidade de Santiago de Compostela, Spain

\footnotetext{
${ }^{a}$ Corresponding author: massimo.barbagallo@ba.infn.it
}

This is an Open Access article distributed under the terms of the Creative Commons Attribution License 4.0, which permits unrestricted use, distribution, and reproduction in any medium, provided the original work is properly cited. 
${ }^{17}$ Paul Scherrer Institut, Villigen PSI, Switzerland

${ }^{18}$ Aristotle University of Thessaloniki, Thessaloniki, Greece

${ }^{19}$ Bhabha Atomic Research Centre (BARC), Mumbai, India

${ }^{20}$ Atominstitut, Technische Universität Wien, Austria

${ }^{21}$ University of York, Heslington, York, UK

${ }^{22}$ Karlsruhe Institute of Technology, Campus Nord, Institut für Kernphysik, Karlsruhe, Germany

${ }^{23}$ Department of Physics, University of Oslo, 0316 Oslo, Norway

${ }^{24}$ University of Vienna, Faculty of Physics, Austria

${ }^{25}$ Dipartimento di Fisica, Università di Bologna and Sezione INFN di Bologna, Italy

${ }^{26}$ Istituto Nazionale di Fisica Nucleare, Laboratori Nazionali di Legnaro, Italy

${ }^{27}$ Agenzia nazionale per le nuove tecnologie, l'energia e lo sviluppo economico sostenibile (ENEA), Bologna, Italy

${ }^{28}$ Istituto Nazionale di Fisica Nucleare, Laboratori Nazionali di Trieste, Italy

${ }^{29}$ Horia Hulubei National Institute of Physics and Nuclear Engineering - IFIN HH, Bucharest Magurele, Romania

${ }^{30}$ European Commission JRC, Institute for Reference Materials and Measurements, 244 Geel, Belgium

${ }^{31}$ Department of Physics and Astronomy, University of Basel, Basel, Switzerland

\begin{abstract}
The study of neutron-induced reactions is of high relevance in a wide variety of fields, ranging from stellar nucleosynthesis and fundamental nuclear physics to applications of nuclear technology. In nuclear energy, high accuracy neutron data are needed for the development of Generation IV fast reactors and accelerator driven systems, these last aimed specifically at nuclear waste incineration, as well as for research on innovative fuel cycles. In this context, a high luminosity Neutron Time Of Flight facility, n_TOF, is operating at CERN since more than a decade, with the aim of providing new, high accuracy and high resolution neutron cross-sections. Thanks to the features of the neutron beam, a rich experimental program relevant to nuclear technology has been carried out so far. The program will be further expanded in the near future, thanks in particular to a new highflux experimental area, now under construction.
\end{abstract}

\title{
1. Introduction
}

In 2012, nuclear energy continued to play an important role in global electricity production. Despite a small reduction of the total generating nuclear power capacity after the accident at the Fukushima Daiichi nuclear power plant, a significant growth, between $35 \%$ and $100 \%$ by 2030 , is foreseen in the use of nuclear energy worldwide [1]. The further exploitation of nuclear energy is limited by several issues affecting current nuclear technology: safety, proliferation, risk of accidents and, especially, the production of radioactive nuclear waste of very long half-life. To overcome these limitations, a new generation of nuclear reactors has been proposed. In particular, in order to minimize nuclear waste to be permanently stored in geological repositories, a closed fuel cycle has to be implemented, aiming at recycling a large fraction of long-lived isotopes, in particular $\mathrm{Pu}$ isotopes and Minor Actinides (such as $\mathrm{Np}$, Am and $\mathrm{Cm}$ ) that constitute the high radiotoxicity long-term component of the nuclear waste. A possible solution now being investigated is represented by the Generation IV fast reactors. By using a nuclear fuel made of U, Pu and minor actinides, such systems would produce energy while reducing the nuclear waste inventory, in particular non-fissile isotopes of very long half life. Another possibility would be the use of dedicated systems for nuclear waste transmutation, in particular subcritical Accelerator Driven Systems. Finally, new fuel cycles, like the Th/U one, are being considered for future use, since they intrinsically produce a much smaller volume of high-radiotoxicity nuclear waste to be stored in geological repositories. 
The development of new technologies in the field of nuclear energy requires improvements in the knowledge of a wide variety of nuclear data. Among them, the most important ones are cross sections for neutron-induced capture, fission and inelastic reactions, which play a key role in the operation of present nuclear reactors, as well as in the design of future ones. High accuracy cross section data for a large number of neutron-induced reactions are therefore required to improve safety and efficiency of current reactors, and for the new developments in the field of nuclear energy. Apart for nuclear technology, accurate neutron cross sections are a fundamental prerequisite for other field of fundamental and applied Nuclear Physics, in particular Nuclear Astrophysics.

Since 2001 a high-luminosity neutron time-of-flight facility, $n \_$TOF, has been operating at CERN with the aim of addressing the needs of nuclear data for basic and applied nuclear Physics [2, 3]. A large International Collaboration, supported by the European Commission within several Framework Programs for Research (Euratom), has carried out so far a vast experimental program on capture, fission and, more recently, on (n,charged particle) reactions. Thanks to the well suited features of the n_TOF neutron beam, such as the high instantaneous neutron flux, the high resolution and the wide energy range covered, from thermal to a few $\mathrm{GeV}$, it has been possible to collect high accuracy and high resolution neutron cross-section data on a variety of isotopes, many of which radioactive. In particular, important results for nuclear technologies have been obtained on isotopes of $\mathrm{U}, \mathrm{Pu}$ and minor actinides with long half life. Recently the construction of a new, high-flux measuring station has started. A 25 times higher fluence relative to the existing experimental area will allow to measure isotopes with short half life, as well as reactions characterized by low cross section. In the following the facility, experimental apparata and some results recently obtained at $\mathrm{n} \_\mathrm{TOF}$ are presented.

\section{The $n \_$TOF facility}

At $n \_$TOF neutrons are produced by means of spallation reactions induced by high energy protons $(20 \mathrm{GeV})$ impinging on a $\mathrm{Pb}$ target. After being partially moderated by an appropriate liquid circulating around the spallation target, also acting as coolant, neutrons propagate through vacuum pipes to the Experimental Area (EAR1), placed at a distance of approximately $200 \mathrm{~m}$. Here the energy of the neutrons is determined by means of the time of flight technique and the reaction products of the neutron interaction with the sample under investigation are detected by suitably chosen detection systems. Thanks to the high peak current and energy of the proton pulse involved in the spallation process, the neutron beam reaching the experimental area is characterized by a very high instantaneous intensity $\left(\sim 10^{6}\right.$ neutrons/pulse), as well as by a wide energy range, from thermal up to $1 \mathrm{GeV}$. The neutron flux measured in EAR1 is shown in Fig. 1 for two different moderator liquids. As a consequences of the long flight-path, the neutron beam is also characterized by an extremely good energy resolution, of the order of $10^{-4}$ in the energy region where most of the isotopes measured present resonances. In addition, the low repetition rate of the proton pulse $(\leq 0.8 \mathrm{~Hz})$ ensures that two consecutive neutron bunches do not overlap.

Finally, a series of beam shaping collimators, iron and concrete shieldings and a sweeping magnet placed along the beam line result in a very low ambient background in EAR1; this aspect, together with the afore-mentioned features of the facility, allow to collect high accuracy data even for reactions of low cross sections or for isotopes available in small quantities. The main advantage of the facility, thanks to the high instantaneous neutron flux, is related to the measurement of radioactive isotopes, such as alpha-emitters minor actinides relevant to the field of nuclear technology.

\section{The experimental setup}

Fission and capture cross section measurements are routinely performed at n_TOF with several experimental setups; similarly, the neutron flux approaching EAR1 is continuously monitored by means 


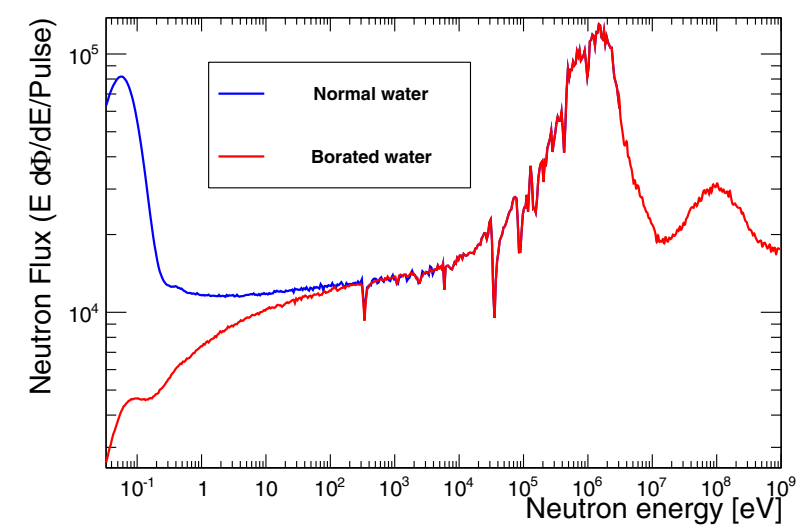

Figure 1. The n_TOF flux measured at the experimental area with two different moderator liquids circulating around the spallation target assembly.

of two different high-transparency detectors while dedicated measurement to determine the absolute neutron flux have been carried out by combining five different detection systems. Such an approach allows to identify and minimize systematic uncertainties associated to each specific detection system and to reach unprecedented accuracy both in the flux determination and in the neutron induced reaction cross sections.

The neutron flux is measured exploiting three different converting reactions, namely ${ }^{6} \operatorname{Li}(\mathrm{n}, \alpha)$, ${ }^{10} \mathrm{~B}(\mathrm{n}, \alpha)$ and ${ }^{235} \mathrm{U}(\mathrm{n}, \mathrm{f})$, in combination with an array of silicon detectors, MicroMegas gas detectors, a calibrated Fission Chamber and a stack of Parallel Plate Avalanche Counters. A careful comparison and combination of the different measurements allows to reach accuracy on the energy-dependence of the neutron flux at the level of a percent [4], which is a fundamental prerequisite of high accuracy results on capture and fission cross sections.

In capture cross section measurements, $\gamma$-rays arising from the deexcitation of the compound nucleus formed in the reaction are detected by means of two different complementary systems: i) an array of $\mathrm{C}_{6} \mathrm{D}_{6}$ liquid scintillators [5] and, ii) a Total Absorption Calorimeter (TAC) [6], made of $40 \mathrm{BaF}_{2}$ crystals. The more complex and costly TAC device is ideal for the measurement of reactions on radioactive isotopes, especially fissionable ones (such as actinides), since it allows to discriminate capture reactions from fission ones on the basis of the total cascade energy detected in each capture event. On the other hand, this system is affected by a large neutron sensitivity, so that scattered neutrons may in some cases represent a large source of background. On the contrary, the low neutron sensitivity of the $\mathrm{C}_{6} \mathrm{D}_{6}$ detectors is an advantage for measurements of isotopes with a high scattering to capture ratio, while the fast response of these detectors makes them more suitable for measuring cross sections in the Unresolved Resonance Region, i.e. from a few $\mathrm{keV}$ up to $1 \mathrm{MeV}$. However, in this case the simplicity of the experimental set-up has the drawback of a more complicated data analysis procedure, which is based on the so-called Pulse Height Weighting Technique (PHWT) [7]. For all these reasons n_TOF data on capture cross-section are provided after comparison and opportune combinations of the results obtained with both detection systems.

Regarding fission cross section measurements, several setups have been used since the beginning at $\mathrm{n} \_$TOF to detect fissions fragments. Typically in the same detector the sample under investigation and a reference sample (namely ${ }^{235} \mathrm{U}$ and ${ }^{238} \mathrm{U}$ ) are placed, so to extract cross sections relative to well known standards, in the so-called ratio method. A standard Fission Ionization Chamber (FIC) made of a stack of parallel plate chambers was used in the first phase of the experimental program [8]. The fissile material is deposited on one electrode. A second fission setup used at $n_{-}$TOF relies on the detection of both fission 
fragments in coincidence, by means of a stack of position-sensitive Parallel Plate Avalanche Counters (PPACs) [9]. Thanks to the coincidence technique it is possible to achieve a very efficient rejection of $\alpha$-particles, which is one of the most important sources of background in this type of measurement. In this case, the fissile material is deposited on very thin aluminum foils placed between two adjacent PPACs. The detectors are position sensitive, so to measure the angular distribution of fission fragments as well.

The third detection system used in fission measurements is MicroMegas detector. MicroMegas is a family of gaseous detectors, initially developed for high-energy experiments, characterized by low noise, high radiation resistance and low mass. For several years, they have been used at n_TOF for measuring the neutron flux, the spatial beam profile and more recently specifically for measurements of neutroninduced fission cross sections. A new version of the Micromegas detector, based on the Micro Bulk technology [10], is currently being used at n_TOF, where further improvements have been introduced leading to an essentially transparent detector. The detector consists of a gas volume acting as a drift region with an electric field of $\sim 1 \mathrm{kV} / \mathrm{cm}$ and a narrow amplification gap ( 25 to $50 \mu \mathrm{m}$ wide) with an electric field of $\geq 10 \mathrm{kV} / \mathrm{cm}$.

\section{Results}

High-quality data on a large number of reactions have been collected at $n_{-}$TOF in two experimental campaigns (2001-2004 and 2009-2012), which have led to improvements in the accuracy of neutron cross-sections for a number of isotopes of interest for nuclear technologies. Some long standing discrepancies between previous results have been solved thanks to the new results. Finally, at n_TOF data have been mostly collected in a wider neutron energy range, compared to previous measurements.

Most of the results obtained so far have already been published and made available in EXFOR [11], the experimental database which collects nuclear data for nuclear technologies and nuclear astrophysics. Among the most important data relevant to nuclear energy, the radiative capture cross-section of ${ }^{232} \mathrm{Th}$ and the fission cross-section of ${ }^{233} \mathrm{U}$ are worth of mentioning, due to their fundamental role in the $\mathrm{Th} / \mathrm{U}$ fuel cycle. In particular, the cross section of the ${ }^{232} \mathrm{Th}(\mathrm{n}, \gamma)$ reaction from $1 \mathrm{eV}$ to $1 \mathrm{MeV}$ has been measured with an unprecedented low uncertainty $(5 \%)[12,13]$, thus allowing to finally clarify a longstanding $40 \%$ discrepancy between previous data in the keV enery region. High accuracy data (i.e. $3 \%$ uncertainty) have been also obtained on the ${ }^{233} \mathrm{U}(\mathrm{n}, \mathrm{f})[14,15]$ from thermal to $20 \mathrm{MeV}$.

Regarding data relevant to transmutation projects or Generation IV fast nuclear reactors, capture and fission cross section of the long-lived minor actinides most abundantly produced in current reactors have been measured. In particular, high quality data, i.e. characterized by unprecedented low uncertainty and high resolution, have been collected on both capture and fission reactions for ${ }^{237} N p[16],{ }^{236} U,{ }^{238} U$, ${ }^{241} \mathrm{Am}$ and ${ }^{243} \mathrm{Am}$, while the the fission cross section of ${ }^{240} \mathrm{Pu},{ }^{242} \mathrm{Pu}$ has recently been measured. One of the best example of the high quality of $n_{-}$TOF data is represented by the results on the fission cross section of ${ }^{245} \mathrm{Cm}$, an extremely difficult measurement due to the high radioactivity of the sample [17]. As a demonstration of the quality of $n \_$TOF data, some results on the ${ }^{241} \mathrm{Am}(\mathrm{n}, \mathrm{f}),{ }^{243} \mathrm{Am}(\mathrm{n}, \mathrm{f})$ and ${ }^{236} \mathrm{U}(\mathrm{n}, \gamma)$ are reported in the following.

Nuclear data on ${ }^{241,243} \mathrm{Am}$, similarly to other minor actinides, play a very important role in the context of transmutation (or incineration) and of longer burn-up cores, not only for the analysis of reactor performance but also for the post-irradiation treatment of spent fuel; for this reason, and due to the lack of reliable experimental data, the fission cross-section of both isotopes has been measured at $\mathrm{n} \_$TOF from thermal to $20 \mathrm{MeV}$. Results for neutron energies in the fast region (above $0.5 \mathrm{MeV}$ ) have been already published $[19,20]$. Figures 2 and 3 show the comparisons between $n \_$TOF data and some of the major evaluated data library, for ${ }^{241} \mathrm{Am}(\mathrm{n}, \mathrm{f})$ and ${ }^{243} \mathrm{Am}(\mathrm{n}, \mathrm{f})$ cross-sections respectively, in the low-energy region, where several resonances are present. 


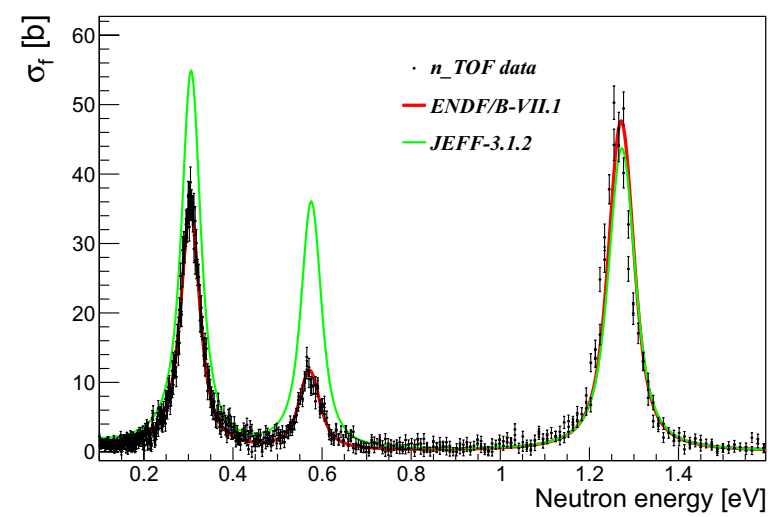

Figure 2. ${ }^{241} \mathrm{Am}(\mathrm{n}, \mathrm{f})$ cross section measured at $\mathrm{n} \_$TOF in comparison with ENDF/B-VII.1 and JEFF-3.1.2 evaluations.

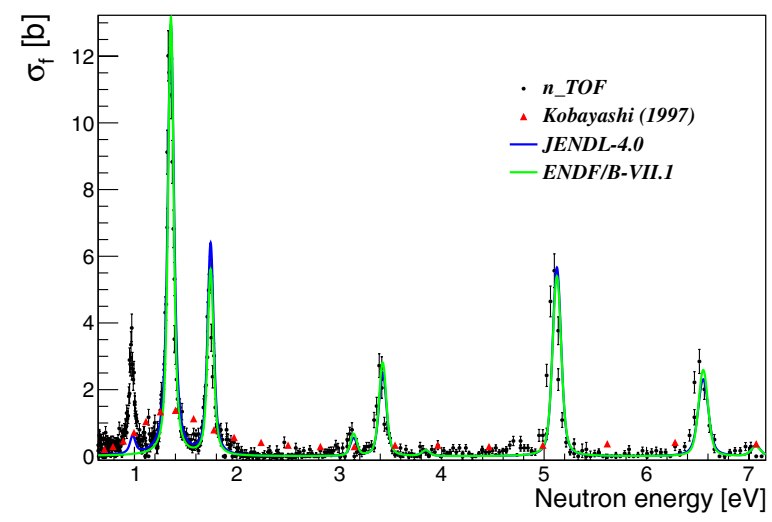

Figure 3. ${ }^{243} \mathrm{Am}(\mathrm{n}, \mathrm{f})$ cross section measured at $\mathrm{n} \_$TOF in comparison with JENDL-4.0 and JEFF-3.1.2 evaluations in the low energy region; also the only previous existing experimental data are reported in the plot.

In the case of ${ }^{241} \mathrm{Am}, \mathrm{n}$-TOF data clearly indicate that JEFF-3.1.2, the european evaluated nuclear data library, needs a revision in the Resolved Resonance Region (RRR) while they essentially confirm ENDF/B-VII.1 evaluation [21].

In the case of ${ }^{243} \mathrm{Am}$ fission cross-section, $\mathrm{n} \_$TOF data are in fair agreement with evaluations, except for some new resonance which has been here identified and which are not contemplated in any of the evaluations. In the same plot, the only previously existing low-resolution data are shown, demonstrating how the unique features of the n_TOF facility can allow to improve considerably the accuracy of cross section data [22].

The radiative capture cross section of ${ }^{236} \mathrm{U}$ has been measured at $\mathrm{n}_{-} \mathrm{TOF}$ both with $\mathrm{C}_{6} \mathrm{D}_{6}$ and TAC detection systems. The ${ }^{236} \mathrm{U}$ plays an important role in nuclear systems, both future and currently operating ones. Specifically, it contributes to the neutron balance in the reactor core and in the fuel composition at equilibrium, in the current reactors based on the $\mathrm{U} / \mathrm{Pu}$ fuel, where it is produced mainly in neutron capture reactions on ${ }^{235} \mathrm{U}$. For future reactors based on the Th/U cycle, ${ }^{236} \mathrm{U}$ plays the same role of ${ }^{242} \mathrm{Pu}$ in the traditional fuel cycle, with a relevant contribution to neutron absorption. For this reason the capture cross-section has been measured for the first time in the whole energy region from thermal energy up to $1 \mathrm{MeV}$ at $\mathrm{n}_{-}$TOF and with the two different detection systems $\left(\mathrm{C}_{6} \mathrm{D}_{6}\right.$ and TAC). 


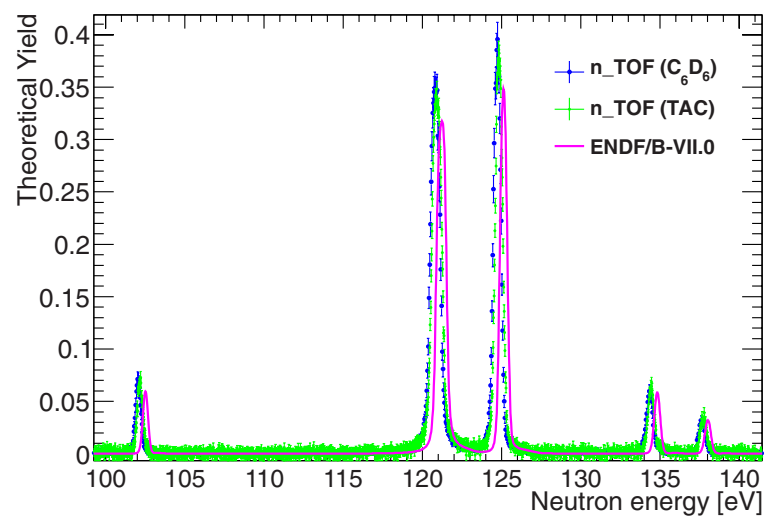

Figure 4. The $n \_$TOF results with both capture setups for ${ }^{236} \mathrm{U}(\mathrm{n}, \gamma)$ cross section measurement in comparison with ENDF/B-VII.0 evaluation in RRR.

In Fig. 4 a preliminary result of the measurements is shown, in comparison also with ENDF/B-VII.0 evaluation: the results of the two detectors, which agree between each other, indicate that the evaluated library need some refinement on both the strength and energy of the resonances. In the URR, on the contrary, n_TOF data confirm evaluated cross sections of major libraries [23].

\section{Conclusions and perspectives}

The $n \_$TOF facility operates at CERN with the aim of addressing the request of high accuracy nuclear data for advanced nuclear energy systems as well as for nuclear astrophysics. Thanks to the features of the neutron beam, important results have been obtained on neutron induced fission and capture cross sections of $\mathrm{U}, \mathrm{Pu}$ and minor actinides, necessary for improving safety and efficiency of current nuclear reactors, and for the development of new generation systems for energy production and nuclear waste transmutation.

A new measurement campaign will soon start with the aim of improving the accuracy of cross sections on more isotopes of interest for nuclear applications, Nuclear Astrophysics, Nuclear Medicine and Basic Nuclear Physics. In particular, new measurements of interest for nuclear technology planned for the near future are the simultaneous capture and fission cross section of ${ }^{233} \mathrm{U}$, the fission of ${ }^{231} \mathrm{~Pa}$ and capture of ${ }^{242} \mathrm{Pu}$.

Moreover, a new beam line is now under construction. It will have a $20 \mathrm{~m}$ flight path and it will be placed on the vertical of the spallation target. As a consequence of the shorter flight path with respect to the existing experimental area, the neutron beam in the new measuring station will be 25 times more intense, with a signal/background ratio expected to be enhanced of a factor of 250 . The new beam line will clearly expand the potentiality of the facility, allowing to measure nuclear reactions not accessible at present. Indeed, although at the expenses of a worse energy resolution, the extremely high instantaneous neutron flux will allow to perform measurements of radioactive isotopes with lifetime as short as a few years. After the commissioning of the new beam line, a rich experimental program is foreseen. In particular, on the side of applications to emerging nuclear technology for energy production, measurements such as ${ }^{238,241} \mathrm{Pu}(\mathrm{n}, \mathrm{f}),{ }^{244} \mathrm{Cm}(\mathrm{n}, \mathrm{f}),{ }^{232} \mathrm{U}(\mathrm{n}, \mathrm{f})$ and ${ }^{245} \mathrm{Cm}(\mathrm{n}, \gamma)$, that cannot be currently performed in any other neutron facility around the world, will finally become feasible at $\mathrm{n} \_$TOF.

The research leading to these results has received funding from the European Atomic Energy Community's (Euratom) Seventh Framework Programme FP7 under the project ANDES, grant agreement n FP7 - 249671. 


\section{References}

[1] Nuclear Technologies Review 2012, GC(56)/INF/3, International Atomic Energy Agency (IAEA)

[2] N. Colonna et al., Energy Environ. Sci. 3, 157 (2010) 1910-1917

[3] F. Käppeler, R. Gallino, S. Bisterzo, and Wako Aoki, Rev. Mod. Phys. 83, 157 (2011)

[4] M. Barbagallo et al., Eur. Phys. J. A 49: 156 (2013)

[5] R. Plag et al., Nucl. Instr. and Meth. A. 496, 425-436 (2003)

[6] C. Guerrero et al., AIP Conf. 1090, 372-375 (2008)

[7] A. Borella et al., Nucl. Instr. and Meth. A. 577, pp. 626-640, 2007

[8] M. Calviani et al., Nucl. Instr. and Meth. A. 594, 220 (2008)

[9] C. Paradela et al., Phys. Rev. C 82, (2010) 044604

[10] I. Giomataris and R. De Oliveira, Patent CEA-CERN, Application Number 09290825.0 (2009)

[11] https://www-nds.iaea.org/exfor/exfor.htm

[12] F. Gunsing et al., Phys. Rev. C 85, (2012) 064601

[13] G. Aerts et al., Phys. Rev. C 73, (2006) 054610

[14] M. Calviani et al., Phys. Rev. C 80, (2009) 044604

[15] F. Belloni et al., Eur. Phys. J. A. 47, 2 (2011)

[16] C. Paradela et al., Phys. Rev. C 82, (2010) 034601

[17] M. Calviani et al., Phys. Rev. C 85, (2012) 034616

[18] C. Guerrero et al., Phys. Rev. C 85, (2012) 044616

[19] F. Belloni et al., Eur. Phys. J. A 47: 160 (2011)

[20] F. Belloni et al., Eur. Phys. J. A 49: 2 (2013)

[21] M. Mastromarco et al., in preparation

[22] M. Mastromarco et al., in preparation

[23] M. Barbagallo et al., in preparation 